\title{
On the existence of Alfvén waves in the terrestrial foreshock
}

\author{
J. P. Eastwood ${ }^{1}$, A. Balogh ${ }^{1}$, E. A. Lucek ${ }^{1}$, C. Mazelle $^{2}$, and I. Dandouras ${ }^{2}$ \\ ${ }^{1}$ Space \& Atmospheric Physics Group, Blackett Laboratory, Imperial College, Prince Consort Rd, London SW7 2BW, UK \\ ${ }^{2}$ CESR/ CNRS, 9, Avenue du Colonel Roche F-31028 Toulouse Cedex 4, France
}

Received: 29 November 2002 - Revised: 2 February 2003 - Accepted: 7 February 2003

\begin{abstract}
The terrestrial foreshock is characterised by the existence of large amplitude ultra low frequency waves. The majority of such waves are observed to be left-handed in the spacecraft frame, but are in fact intrinsically right-handed and have been identified as fast-magnetosonic waves. More rarely observed are waves that are right-handed in the spacecraft frame. Cluster four spacecraft observations of such waves are presented and analysed using multi-spacecraft techniques; in particular the $k$-filtering/wave telescope technique is used. The waves are found to be left-handed and propagating sunwards in the plasma rest frame, and are, therefore, identified as Alfvénic. The convection of the waves anti-sunward in the solar wind flow causes the observed polarisation to be reversed. Generation mechanisms are discussed.
\end{abstract}

Key words. Interplanetary physics (MHD waves and turbulence; planetary bow shocks) - Space plasma physics (wave particle interactions)

\section{Introduction}

It is now well understood that of all the ways in which a collisionless plasma shock is different from its neutral gas counterpart, one of the most striking is that a small proportion of the inflowing fluid is reflected at the shock to return upstream (e.g. Fuselier, 1994; Asbridge, 1968). The combination of the inflowing plasma and the backstreaming particles upstream of the shock is unstable to a number of different wave particle interactions (e.g. Gary, 1993). Momentum and energy are transferred from the beam to the bulk flow, via the local generation of Ultra Low Frequency (ULF) waves (Gary, 1978; Bonifazi et al., 1980, Bame et al., 1980). The region of space upstream of a collisionless shock characterised by such particle distributions and wave activity is known as the foreshock.

Correspondence to: J. P. Eastwood

j.p.eastwood@ic.ac.uk
Although the beam particles are sufficiently energetic to escape upstream and never re-encounter the shock, the waves propagate at speeds much less than the upstream flow velocity, and are, therefore, convected back into the shock regardless of their propagation direction. The large amplitude of the waves can directly affect the shock itself by causing $\theta_{B n}$, the angle between the shock normal and the magnetic field to vary (Greenstadt and Mellott, 1985). Furthermore, the waves can be transmitted through the shock, where in the context of the terrestrial bow shock, they may influence magnetospheric dynamics.

The majority of upstream ULF waves are left-handed in the spacecraft frame of reference. This is defined with respect to the magnetic field. These waves are intrinsically right-handed (i.e. fast-magnetosonic) and are attempting to propagate upstream, in the direction of the beam that generated them. However, as explained above, they are convected back towards the shock by the super-magnetosonic solar wind (Le and Russell, 1994; Eastwood et al., 2002), causing the observed reversal in polarisation.

Upstream waves that are right-handed in the spacecraft frame have also been observed. Hoppe and Russell (1983) identified in the ISEE dual spacecraft data set one interval of multi-frequency shocklets/waves which could be analysed with two spacecraft. They concluded that since these waves had both left- and right-handed polarisations, the waves were a combination of Alfvénic and magnetosonic fluctuations. In reviews of foreshock research in the mid 1990s, it was clearly stated that compared to the waves observed to be left-handed in the spacecraft frame, the right-handed waves were not well understood (Le and Russell, 1994; Greenstadt, 1995).

Theoretically, two possibilities exist for the intrinsic mode of waves observed to be right-handed. The first is that they are Alfvénic (left-handed) in nature and attempting to propagate upstream, but are then convected downstream reversing the observed polarisation. In this case they would most likely be generated by a resonant instability, produced by hot backstreaming ions (Gary, 1985). The second is that they are fast-magnetosonic (right-handed) in nature and propagating 
Cluster Orbit year 2002 day 34 x-y GSE plane. Tetrahedron x200

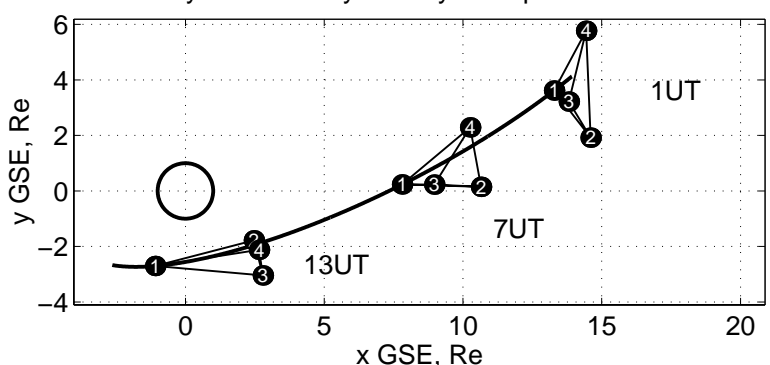

Cluster Orbit year 2002 day $34 \mathrm{x}-\mathrm{z}$ GSE plane. Tetrahedron x200

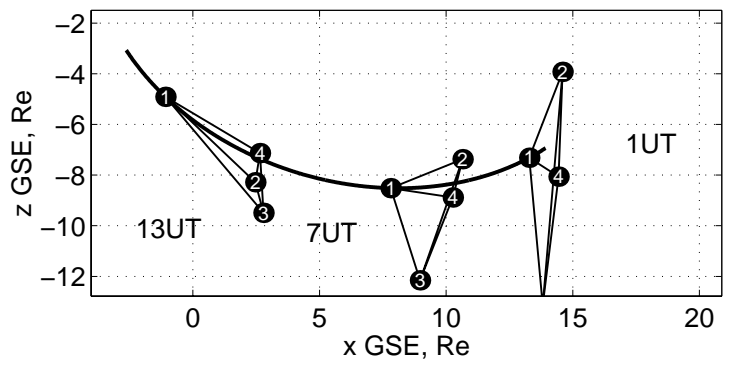

Fig. 1. Cluster orbit on day 34, 3 February 2002. Projections of the Cluster 1 orbit in the $x-y$ (top) and $x-z$ (bottom) GSE plane are shown. The spacecraft tetrahedron, magnified $\times 200$, is shown at 01:00 UT, 07:00 UT and 13:00 UT.

downstream, in which case the Doppler shift has no effect. The proposed candidate mechanism for their generation is the non-resonant firehose instability (Sentman et al., 1981). In both cases, the energy source causing the waves to grow is the back-streaming particle beam. In the case of Alfvénic fluctuations, the waves propagate parallel to the beam. In the case of fast-magnetosonic fluctuations, the waves propagate anti-parallel to the beam.

More recently, Blanco-Cano and Schwartz (1997) used single spacecraft AMPTE-UKS observations to determine the properties of foreshock ULF waves. The data were used to calculate transport ratios, which were then compared to the theoretical transport ratios of waves generated by cyclotron resonances as modelled by linear kinetic theory using "typical observed values". Intervals of right-handed waves were identified as being Alfvénic. However, non-resonant interactions were not considered and a number of caveats applied. It was not possible to evaluate the moments of the diffuse ion distributions associated with the observed waves and it was noted that multi-spacecraft analysis was required for a more complete description.

It has been proposed that the wave field plays an important role in the structure of the shock itself and that as the waves approach the shock, they grow and steepen, forming so-called Short Large Amplitude Magnetic Structures (SLAMS). These structures then coalesce to form the shock surface (Schwartz and Burgess, 1991). However, many details of the mechanism remain unresolved. In particular, it is noted that SLAMS are typically observed to be right-handed in the spacecraft frame of reference (Schwartz et al., 1992) and are propagating upstream into the solar wind at super- magnetosonic speeds, but are still convected downstream, since their propagation speed is less than the solar wind speed. SLAMS are, therefore, left-handed in the plasma rest frame. If they are sourced from the ULF wave field, one might propose that they should be associated with waves exhibiting similar polarisation properties.

With the launch of the 4 spacecraft Cluster mission, new data is now available to address these and other questions concerning the dynamics of the shock and foreshock, as part of its overall goal to study magnetospheric dynamics. Here, we report on Cluster observations of a period of remarkably well-defined foreshock wave activity, where the waves are observed to be right-handed in the spacecraft frame of reference.

In Sect. 2, an overview of the data is presented. In Sect. 3, a wave analysis technique based on cross correlation timing techniques is applied to the data (Eastwood et al., 2002). In Sect. 4 , the $k$-filtering/wave telescope technique is applied to the data. This technique has received considerable theoretical attention and is one of the three main multi-spacecraft analysis techniques (the others being the discontinuity analyser and the curlometer). In Sect. 5, the results are drawn together, and possible generation mechanisms are discussed.

\section{Overview of data}

The data analysed in this paper were taken on 3 February 2002 (day 34). At this time, the mission phase was such that the separation of the spacecraft was $\sim 100 \mathrm{~km}$. Figure 1 shows the orbit of Cluster on this day between 00:00:00 UT and 14:00:00 UT. The top panel is a projection in the $x-y$ Geocentric Solar Ecliptic (GSE) plane, and the bottom panel a projection in the $x-z$ GSE plane. The orbit of Cluster 1 is shown. The spacecraft tetrahedron is shown at 01:00:00 UT, 07:00:00 UT and 13:00:00 UT, magnified by a factor of 200 . At the start of the day, Cluster was located in the solar wind, on the dusk flank of the magnetosphere, and during this interval, the spacecraft descended south under the ecliptic.

Each Cluster spacecraft carries an identical Flux-Gate Magnetometer (FGM) instrument to measure the properties of the local magnetic field (Balogh et al., 2001). The three panels on the left-hand side of Fig. 2 show the magnetic field measured by the FGM instrument on board Cluster 1 for the interval 00:00:00 UT-14:00:00 UT, day 34, 2002, in GSE polar coordinates $\left(\Phi_{B}\right.$ is the angle relative to the $x$ axis in the ecliptic plane, and $\theta_{B}$ is the angle out of the ecliptic). The data is shown at $4 \mathrm{~s}$ (spin) resolution; differences between the four spacecraft are indistinguishable on this scale. Cluster was initially in the solar wind, entering the foreshock just before 04:00:00 UT. At just before 05:00:00 UT, Cluster encountered the bow shock, which exhibited an extended and disordered structure. The spacecraft entered the magnetosphere at approximately 09:15:00 UT.

The four panels on the right-hand side of Fig. 2 show the magnetic field measured by the Cluster 1 FGM instrument for the interval 04:00:00 UT-04:10:00 UT, at a resolution of 


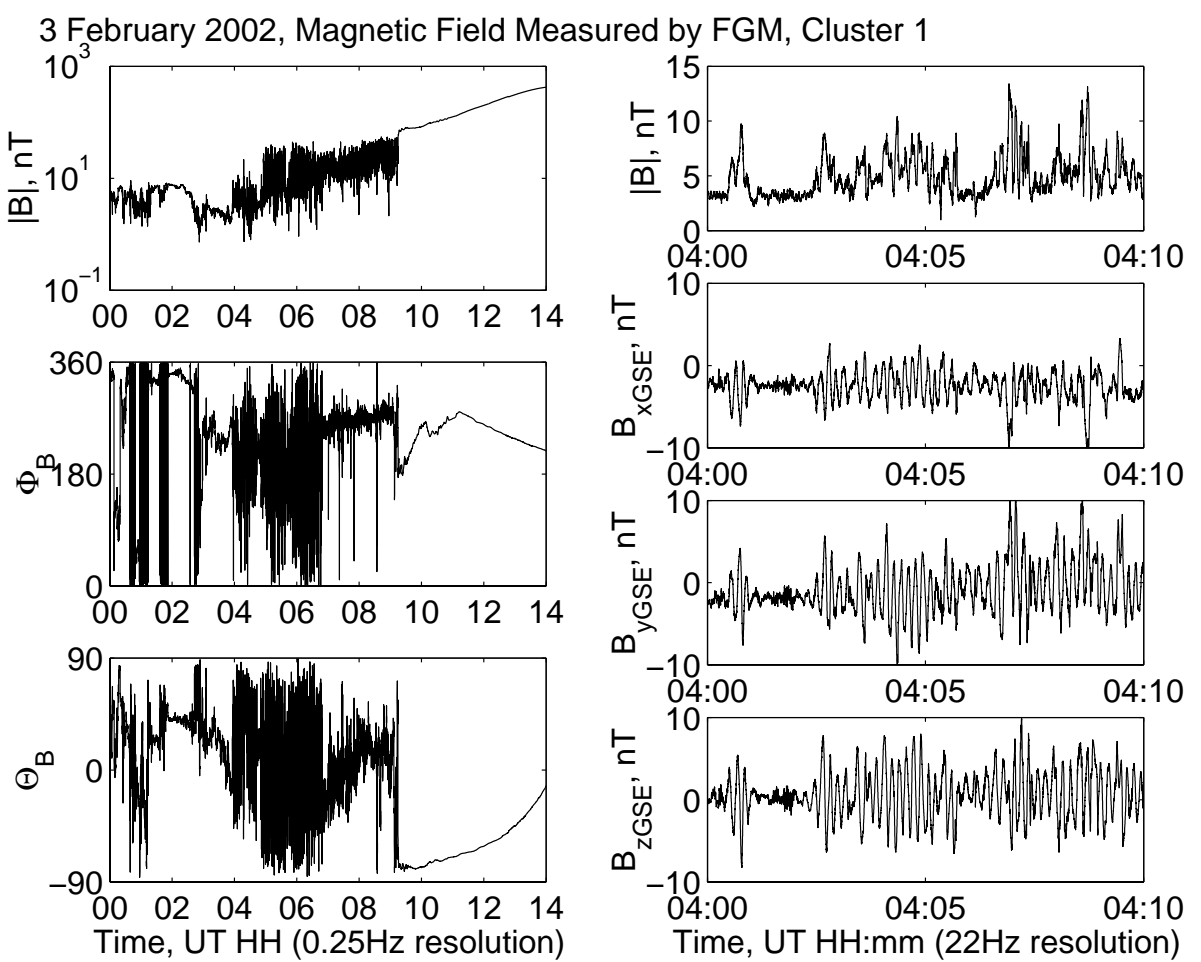

Fig. 2. FGM Cluster 1 data for day 34, 3 February 2002. The three left-hand panels show the data for the whole interval in GSE polar coordinates. The four right-hand panels show the interval 04:00 UT-04:10 UT. The time series are $|B|$ (top), $B_{x}, B_{y}, B_{z}$ (bottom).

22 vectors/s. From top to bottom on the right-hand side, the time series of $|B|, B_{x}, B_{y}$ and $B_{z}$ are displayed. During this interval, the orientation of the magnetic field was such that the spacecraft were magnetically connected to the shock and located in the foreshock. The time series recorded by the FGM instruments on the other three spacecraft are identical on this scale, but offset in time, due to the motion of the phase fronts in the spacecraft frame. The waves are exceptionally well defined, with very low turbulent noise. Therefore, despite the fact that the time offsets between the four time series are fairly small, $(\sim 1 / 3$ s given the spacecraft separation $)$, the definition of the wave form allows the analysis to proceed. During the interval, the solar wind speed was of the order of $400 \mathrm{kms}^{-1}$.

The polarisation of the waves in the spacecraft frame can be identified by applying Minimum Variance Analysis (MVA) to the magnetic field time series. For circularly polarised waves, the minimum variance direction may be identified, with a $180^{\circ}$ ambiguity, as the direction of propagation of the wave (e.g. Song and Russell, 1999). MVA was applied to a short interval of wave activity (3 cycles) 04:04:00 UT04:04:30 UT at each of the four spacecraft and the data was transformed from GSE coordinates into the minimum variance coordinate system. The results are summarised in Fig. 3. The top four panels show hodograms derived from each of the four spacecraft time series. In each case, the minimum variance direction is directed out of the page. The symbol in the bottom right corner of each plot shows the direction of the average magnetic field relative to the minimum variance direction. The cross signifies that the average field is directed into the page. The solid dot on the trace indicates the start point of the hodogram. It can be seen that for all the spacecraft, the trace follows a clockwise path. Since the average field is directed into the page, this means that these waves are right-handed with respect to the magnetic field in the spacecraft frame of reference.

The bottom two panels show the orientation of the four minimum variance directions (solid lines) and the average magnetic field direction (dotted line) in GSE. The four solid lines are indistinguishable, indicating that the MVA applied to each of the spacecraft gives very similar results. It can also be seen that the waves are not field-aligned, according to this analysis. The average magnetic field is in the $-x /-y$ direction, which, given the location of Cluster on the dusk flank of the bow shock, meant that Cluster was magnetically connected to the bow shock, as mentioned above.

Both single and dual spacecraft analysis fundamentally rely on MVA to determine the orientation of the waves. However, the method is limited to waves that are strongly circularly polarised so that degeneracy between the intermediate and minimum variance directions is avoided (Song and Russell, 1999). An important point also to bear in mind is that although the MVA results are consistent between the four spacecraft, it is possible that they each suffer the same systematic errors and that the MVA result does not necessarily correspond to the true wave orientation. However, the usefulness of MVA in revealing the polarisation of the waves is unaffected. 

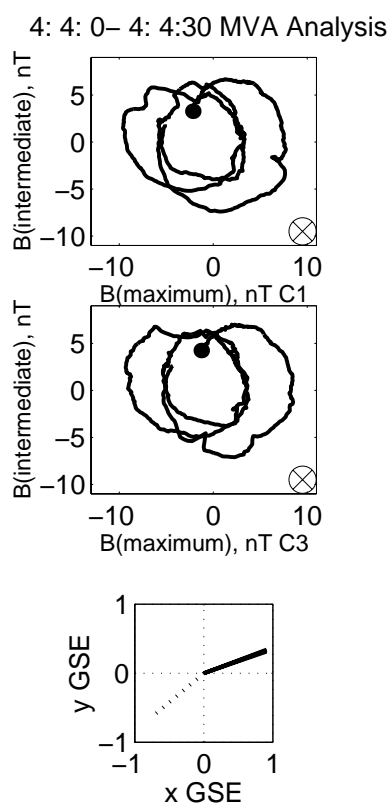

Fig. 3. MVA analysis for the interval 04:04:00 UT-04:04:30 UT. The top four panels are hodograms derived from each of the four spacecraft. The cross symbol indicates that the average magnetic field is directed into the page. The dot on the trace indicates the start point. The bottom two points show the orientation of the four minimum variance directions (solid) and the magnetic field (dotted) projected into the $x-y$ (left) and $x-z$ (right) GSE planes.

In single spacecraft data analysis, very often it is ambiguous as to whether the observed variations are spatial or temporal in nature. In order to resolve this satisfactorily, at least four spacecraft are required to sample the plasma. Essentially, one spacecraft can be considered to make reference measurements, while the other three make simultaneous comparative measurements in linearly independent directions. Consequently, four spacecraft are sufficient to characterise the local spatial structure of the plasma at any moment. A number of techniques derived from this basic principle are described in the book edited by Paschmann and Daly (1998) and references therein. In particular, of most interest in this work is the $k$-filtering/wave telescope technique (Pinçon and Motschmann, 1998). This technique is ideally suited to the analysis of the data presented here. However, before applying this technique, a less computationally intensive method was used to make a preliminary analysis.

\section{Analysis of wave modes}

Before applying the $k$-filtering/wave telescope technique, a simpler method was used to analyse the data. $K$-filtering is designed to resolve waves of the same frequency propagating in different directions with different wave numbers. However in this case, the symmetry of the problem is broken by the magnetic field and the fact that the wave growth is anisotropic. In particular, waves are expected to grow (approximately) aligned to the field. A less computationally intensive technique has been developed by taking this into account, which has been applied to the analysis of fast-magnetosonic foreshock waves (Eastwood et al., 2002; Mazelle et al., 2002). The method is briefly restated here.

It is assumed that there is a single wave mode, and that the phase front is planar on the scale of the spacecraft separation. It is also assumed that the phase front is moving at constant speed. If spacecraft $\alpha$ located at $\boldsymbol{R}_{\alpha}$ encounters a phase front at $t_{\alpha}$, then given a plane wave with frame invariant unit direction of propagation $\hat{\boldsymbol{k}}$, and spacecraft frame phase speed $\boldsymbol{v}_{p h}$, we can construct the following equations.

$$
\begin{aligned}
& \left(\boldsymbol{R}_{\beta}-\boldsymbol{R}_{1}\right) \cdot \hat{\boldsymbol{k}}=v_{p h}\left(t_{\beta}-t_{1}\right) \quad \beta=2,3,4, \\
& \boldsymbol{v}_{p h}=v_{p h} \cdot \hat{\boldsymbol{k}} .
\end{aligned}
$$

By inverting these equations $\hat{\boldsymbol{k}}$ and $v_{p h}$ are found. The phase speed in the plasma frame, $v_{p l}$, is then calculated by subtracting $\boldsymbol{v}_{s w} \cdot \hat{\boldsymbol{k}}$ from $v_{p h}$. The time lags are calculated by cross-correlating the spacecraft time series. This automatically centres on the largest features in the data, and washes out any effects due to smaller sub-cycle variations. If necessary, the data can be filtered to remove high frequency noise (of physical origin), although this should be done with care, particularly when analysing short intervals of data. In this case, data filtering was not carried out, given the very low noise level in the data.

This technique was applied to the interval 04:02:30 UT04:10:00 UT. In this analysis solar wind moments constructed from measurements taken by the HIA/CIS instrument on board Cluster 1 were used (Rème et al., 2001). The moments of the full 3-D distribution are calculated on board, and are downlinked at 4-s resolution. In the plasma rest frame, these waves were found to propagate along $\hat{\boldsymbol{k}}=$ $(0.930 .19-0.32)$ with a phase speed of $33 \mathrm{kms}^{-1}$, at an angle of $\sim 2^{\circ}$ to the field. Furthermore, the wavelength of the waves was estimated to be $3400 \mathrm{~km}$.

The Alfvén speed was estimated to be $26 \mathrm{kms}^{-1}$ and the sound speed $104 \mathrm{kms}^{-1}$. On the basis of this analysis, the waves are attempting to propagate upstream, but are convected downstream in the solar wind flow. Their intrinsic polarisation is, therefore, left-handed, and the waves are identified as Alfvénic.

As a consistency check, the same analysis was applied to shorter intervals of data. The interval 04:02:30 UT04:10:00 UT was split into ten $45 \mathrm{~s}$ intervals. Given that the period of the waves was $10 \mathrm{~s}$, each interval contained approximately 4 cycles. The results of this analysis are shown in Table 1.

For each interval, the phase speed in the plasma rest frame and the direction of propagation were calculated. In addition, the Alfvén and sound speeds were estimated. The conclusion ( $\mathrm{F}$ = fast magnetosonic, $\mathrm{A}=$ Alfvénic) was based on considerations of the propagation speed and the polarisation. Of the 10 intervals, 7 were consistent with the conclusion that the 
Table 1. Results of the cross-correlation wave analysis technique applied to ten consecutive $45 \mathrm{~s}$ intervals between 04:02:30 UT and 04:10:00 UT. $v_{p l}$ is the phase speed in the plasma frame

\begin{tabular}{ccccccc}
\hline Start Time & $v_{p l}$ & $\hat{\boldsymbol{k}}$ & $\theta_{B k}$ & $V_{a}$ & $C_{S}$ & Conclusion \\
\hline $04: 02: 30$ & -145 & {$[0.680 .71-0.16]$} & 159 & 27 & 92 & $\mathrm{~F}$ \\
$04: 03: 15$ & -193 & {$[0.740 .64-0.20]$} & 171 & 27 & 92 & $\mathrm{~F}$ \\
$04: 04: 00$ & 59 & {$[0.900 .22-0.39]$} & 147 & 30 & 95 & $\mathrm{~A}$ \\
$04: 04: 45$ & 38 & {$[0.890 .16-0.42]$} & 156 & 24 & 97 & $\mathrm{~A}$ \\
$04: 05: 30$ & 41 & {$[0.91-0.36-0.21]$} & 142 & 23 & 98 & $\mathrm{~A}$ \\
$04: 06: 15$ & 69 & {$[0.92-0.01-0.38]$} & 173 & 32 & 104 & $\mathrm{~A}$ \\
$04: 07: 00$ & 31 & {$[0.93-0.26-0.27]$} & 162 & 24 & 105 & $\mathrm{~A}$ \\
$04: 07: 45$ & -38 & {$[0.96-0.16-0.24]$} & 174 & 27 & 111 & $\mathrm{~F}$ \\
$04: 08: 30$ & 7 & {$[0.800 .55-0.24]$} & 127 & 39 & 117 & $\mathrm{~A}$ \\
$04: 09: 15$ & 39 & {$[0.96-0.050 .29]$} & 144 & 27 & 124 & $\mathrm{~A}$ \\
\hline
\end{tabular}

waves are Alfvénic and propagating upstream in the plasma rest frame, in agreement with the analysis of the whole interval.

Having analysed the waves using this method, the data were then analysed using the $k$-filtering/ wave telescope technique. This is a powerful and widely applicable technique designed to reveal the simultaneous existence of multiple wave modes in field time series.

\section{$4 k$-filtering/wave telescope analysis of the wave interval}

Before the launch of Cluster, significant efforts were made in the development of multi-spacecraft analysis techniques. Dunlop et al. (1988) were the first to propose a technique based on multi-spacecraft analysis to determine the properties of waves. In this method, termed the wave telescope, the properties of waves would be determined by examining the relative phases of signals at each of the spacecraft. Neubauer et al. (1990) and Neubauer and Glassmeier (1990) further developed this idea by proposing the use of linear filter theory, in combination with the known theoretical properties of the wave modes, to decompose 4 spacecraft wave measurements into a series of superposed plane waves.

Fundamentally, the aim of the analysis is to determine how the field energy density is distributed as a function of $\omega$ and $\boldsymbol{k}$ : peaks in this distribution correspond to perturbations of particular frequency and wave number. Given an infinite number of measurement points in space, Fourier inversions would allow the $\omega-\boldsymbol{k}$ domain to be mapped completely, and the distribution of field energy could then be determined absolutely. However, in the case of Cluster, we are limited to 4 measurement points in space.

A statistical formalism has been developed that uses the data from a finite number of sampling points to estimate the distribution of field energy density as a function of $\omega$ and $\boldsymbol{k}$; this was originally developed in response to problems in seismology and sonar (e.g. Pillai, 1989 and references therein). Pinçon et al. (1990) applied these techniques to the problem of plasma waves, developing a technique which included the variations of both the electric and magnetic fields. This was further modified by Motschmann et al. (1996), who adapted the method to use only the magnetic field data. We do not wish to discuss the mathematics of the method here; the interested reader is directed to Pinçon and Motschmann (1998) and references therein. We simply note that the four spacecraft time series can be combined to estimate the distribution of field energy density as a function of $\omega$ and $\boldsymbol{k}$. Usually, a specific frequency is identified by Fourier transforming the data and the $\boldsymbol{k}$ space is then scanned to find the maxima of the distribution. The method has been tested extensively, and it has been found that up to 7 different superimposed waves, each with the same frequency, can be distinguished (Motschmann et al., 1996).

The $k$-filter/wave telescope technique was applied to the interval 04:02:30 UT-04:10:00 UT, using data from all four spacecraft at $22-\mathrm{Hz}$ resolution. The data were Fourier transformed and the frequency carrying the most power was estimated to be $0.11 \mathrm{~Hz}$ in the spacecraft frame.

Using the technique, the distribution of field energy in the $\boldsymbol{k}$ space for this frequency was calculated. Small-scale structure in the magnetic field time series would correspond to powers at much higher frequencies, and so this technique concentrates solely on the dominating low frequency component that is of interest. A single maximum in the distribution was found at $\boldsymbol{k}=[-0.002-0.0006670 .00033] \mathrm{km}^{-1}$. Figure 4 shows this distribution in the $k_{z}=3 \times 10^{-4} \mathrm{~km}^{-1}$ plane. The peak in the field energy density is clearly visible at the appropriate location in the $k_{x}-k_{y}$ plane.

The unit direction of propagation was calculated to be $(-0.937-0.3130 .156)$, and the phase speed of the wave in the spacecraft frame was calculated to be $(2 \pi f) /|\boldsymbol{k}|=$ $324 \mathrm{kms}^{-1}$. To determine the phase speed in the plasma rest frame, the Doppler shift $\boldsymbol{v}_{s w} \cdot \hat{\boldsymbol{k}}$ must again be included, giving a phase speed in the plasma rest frame of $-23 \mathrm{kms}^{-1}$. The wave is propagating upstream in the plasma rest frame but is swept downstream in the solar wind flow. It must, therefore, be left-handed in the plasma rest frame, in order to be observed as right-handed in the spacecraft rest frame. 

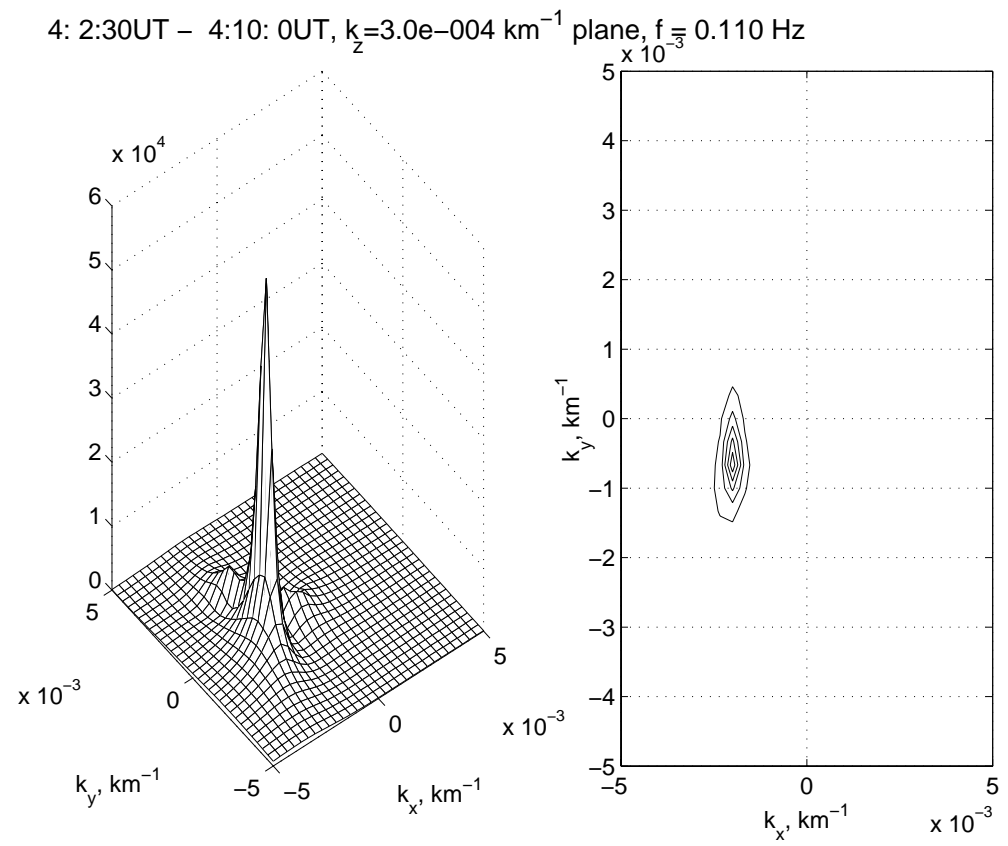

Fig. 4. Results of the $k$-filtering/wave telescope analysis for the interval 04:02:3 0UT-04:10:00 UT, at a frequency of $0.11 \mathrm{~Hz}$. The plot is shown in the $k_{z}=3 \times 10^{-4} \mathrm{~km}^{-1}$ plane. The peak in the power is seen at the appropriate location in $k_{x}$ and $k_{y}$.

Table 2. Summary of results for the interval 04:02:30 UT-04:10:00 UT

\begin{tabular}{|c|c|c|}
\hline & Cross-correlation & $k$-filter/wave telescope \\
\hline Plasma Frame Phase Speed $\left(\mathrm{kms}^{-1}\right)$ & 33 & 23 \\
\hline Direction of Propagation, $\hat{\boldsymbol{k}}$ & {$\left[\begin{array}{lll}0.93 & 0.19-0.32\end{array}\right]$} & {$\left[\begin{array}{llll}0.94 & 0.31 & 0.16\end{array}\right]$} \\
\hline Wavelength $(\mathrm{km})$ & 3400 & 2900 \\
\hline $\begin{array}{l}\text { Plasma Frame Frequency }\left(\mathrm{s}^{-1}\right) \\
V_{a}=26 \mathrm{kms}^{-1}, C_{s}=104 \mathrm{kms}^{-1}, \Omega=0.26 \mathrm{~s}^{-1}\end{array}$ & 0.05 & 0.06 \\
\hline
\end{tabular}

The results of the $k$-filter/wave telescope are consistent with the cross-correlation timing method.

\section{Discussion}

The cross-correlation wave analysis technique and the $k$ filtering/wave telescope technique have been applied to 4 spacecraft Cluster observations of ULF waves upstream of the bow shock in the foreshock. The results are summarised in Table 2. Note that the angular ion gyrofrequency is quoted.

It is clear that the two techniques give consistent results despite their different approaches. On the basis of these results, it is concluded that these waves are Alfvénic in nature.

Of immediate interest is the mechanism by which these waves are generated. It is proposed that the left-hand resonant ion beam electromagnetic instability is responsible. In this mechanism, the left-handed wave resonates with ions moving opposite to that with the net drift. However, for the growth rate to be significant, the beam thermal speed must be at least as great as the beam drift speed.

A number of different types of beam distribution have been observed in the foreshock (e.g. Fuselier, 1994, provides a comprehensive review of the properties of energetic ions in the terrestrial foreshock). Of particular relevance to this work are the so-called diffuse ion distributions, characterised by relatively low drift velocities, and high temperatures (i.e. a large spread in the pitch angle distribution), with a high energy tail to the distribution. Of all types of beam observed in the foreshock, the diffuse beam is the best candidate for generating left-handed waves via resonances.

In order to understand this further, it is necessary to look at the distributions associated with the observed waves. Figure 5 shows energy spectrograms produced from measurements taken by the CIS instrument on board spacecraft 3 between 04:00 UT and 04:10 UT, the same interval as in Fig. 2. The top panel (panel 1) shows the spacecraft mode. During this interval, the instrument mode was such that the HIA instrument sampled the plasma equally in all directions (for details of the modes and implications for the data products the reader is advised to consult Rème et al., 2001). Panels 2, 3,4 and 5 show the fluxes for all ion species in the sunward, duskward, earthward and dawnward look directions, as measured by HIA. In panel 2, the solar wind corresponds to the continuous flux at $\sim 1000 \mathrm{eV}$. It can be seen that after 04:02:30 UT, there are significant fluxes of ions in all 


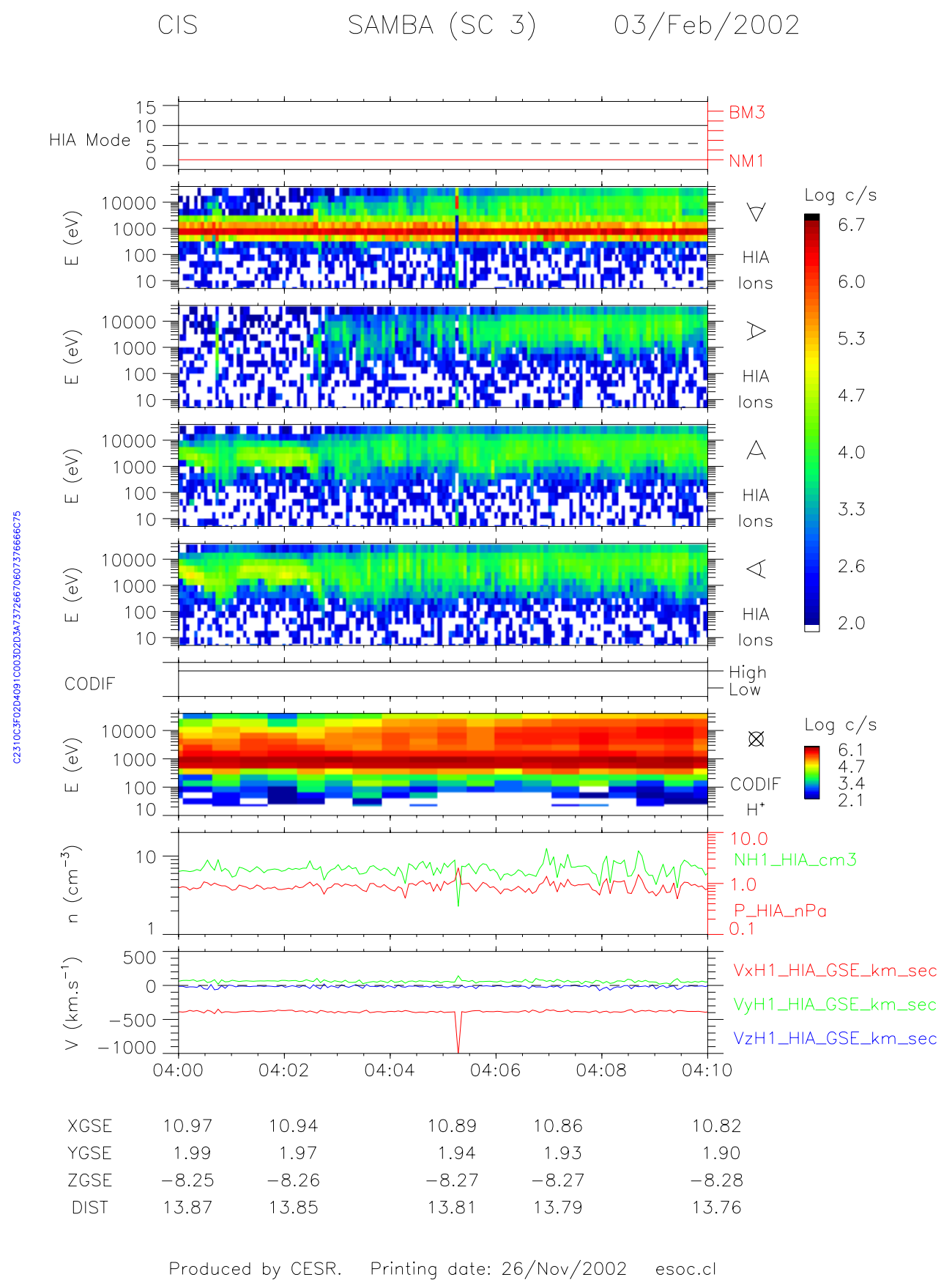

Fig. 5. Energy spectrogram from CIS s/c 3, 04:00 UT-04:10 UT, day 34, 2002. Panels 2, 3, 4 and 5 show the fluxes for all ion species in the sunward, duskward, earthward and dawnward look directions. The waves were observed after 04:02:30 UT, and are associated with a diffuse energetic ion population.

look directions at energies extending to above $10 \mathrm{keV}$. This is a characteristic signature of diffuse ion distributions, and, therefore, is consistent with the overall picture we have outlined. Future work is planned to determine the properties of the diffuse beam, and to determine the growth rates of the left handed resonant instability.

In this work, we have analysed the properties of the signal which carries the vast majority of the power. The cross- correlation timing technique washes out small-scale variations, since the timing is based on the largest features in the time series. The $k$-filtering/wave telescope treats the signal as a Fourier superposition of waves, and the Fourier component carrying the largest power has been analysed. A study of sub-cycle variations, which may reveal small-scale structure at higher frequencies, is currently underway.

To conclude, an interval of waves exhibiting right-handed 
polarisation in the spacecraft frame has been observed by Cluster. This data has been analysed using two different multi-spacecraft techniques. The results of both analyses suggest that these waves are intrinsically left-handed, attempting to propagate upstream but carried downstream in the solar wind flow, and, therefore, Alfvénic. Theoretically, such waves can be generated by sufficiently hot ion beams, corresponding to diffuse ion distributions in the terrestrial foreshock. Examination of energy spectrograms reveals that these waves are observed in association with diffuse ion distributions. Further work is planned to compare the theoretical growth rates with the observed properties of the ion distributions.

Acknowledgements. This work is supported by PPARC.

Topical Editor T. Pulkkinen thanks G. Paschmann and A. Barnes for their help in evaluationg this paper.

\section{References}

Asbridge, J. R., Bame, S. J., and Strong, I. B.: Outward Flow of Protons from the Earth's Bow Shock, J. Geophys. Res., 73, 57775782, 1968.

Balogh, A., Carr, C. M., Acuña, M. H., Dunlop, M. W., Beek, T. J., Brown, P., Fornaçon, K.-H., Georgescu, E., Glassmeier, K.H., Harris, J., Musmann, G., Oddy, T., and Schwingenschuh, K.: The cluster magnetic field investigation: overview of inflight performance and initial results, Ann. Geophysicae, 19, 1207-1217, 2001.

Bame, S. J., Asbridge, J. R., Feldman, W. C., Gosling, J. T., Paschmann, G., and Sckopke, N.: Deceleration of the Solar Wind Upstream from the Earth's Bow Shock and the Origin of Diffuse Upstream Ions, J. Geophys. Res., 85, 2981-2990, 1980.

Blanco-Cano, X. and Schwartz, S. J.: Identification of lowfrequency kinetic wave modes in the Earth's ion foreshock, Ann. Geophysicae, 15, 273-288, 1997.

Bonifazi, C., Moreno, G., Lazarus, A. J., and Sullivan, J. D.: Deceleration of the Solar Wind in the Earth's Foreshock Region: Isee 2 and Imp 8 Observations, J. Geophys. Res., 85, 6031-6038, 1980.

Dunlop, M. W., Southwood, D. J., Glassmeier, K.-H., and Neubauer, F. M.: Analysis of multipoint magnetometer data, Adv. Space. Res., 8, (9)273-(9)277, 1988.

Eastwood, J. P., Balogh, A., Dunlop, M. W., Horbury, T. S., and Dandouras, I.: Cluster observations of Fast Magnetosonic Waves in the Terrestrial Foreshock, Geophys. Res. Lett., 29(22), 2046, doi:10.1029/2002GL015582, 2002.

Fuselier, S. A.: Suprathermal Ion Upstream and Downstream from the Earth's Bow Shock, in: Solar Wind Sources of Ultra-LowFrequency Waves, AGU Monograph 81, Washington D.C., 107119, 1994.

Gary, S. P.: The Electromagnetic Ion Beam Instability and Energy Loss of Fast Alpha Particles, Nucl. Fus., 18, 327-334, 1978.

Gary, S. P.: Electromagnetic Ion Beam Instabilities: Hot Beams at Interplanetary Shocks, Astrophys. J., 88, 342-352, 1985

Gary, S. P.: Theory of Space Plasma Microinstabilities, Cambridge University Press, Cambridge, 1993.

Greenstadt, E. W. and Mellott, M. M.: Variable field-to-normal shock-foreshock boundary observed by isee-1 and -2, Geophys. Res. Lett., 12, 129-132, 1985.
Greenstadt, E. W., Le, G., and Strangeway, R. J.: ULF Waves in the Foreshock, Adv. Space Res., 15, (8/9)71-(8/9)84, 1995.

Hoppe, M. M. and Russell, C. T.: Plasma Rest Frame Frequencies and Polarisations of the Low Frequency Upstream Waves: ISEE 1 and 2 Observations, J. Geophys. Res., 88, 2021-2028, 1983.

Le. G. and Russell, C. T.: The Morphology of ULF waves in the Earth's Foreshock, in: Solar Wind Sources of Ultra-LowFrequency Waves, AGU Monograph 81, Washington D.C., 8798, 1994.

Mazelle, C., Meziane, K., Quéau, Le, D., Wilber, M., Eastwood, J. P., Rème, H., Sauvaud, J. A., Bosqued, J. M., Dandouras, I., McCarthy, M., Kistler, L. M., Klecker, B., Korth, A., BavassanoCattaneo, M. B., Lundin, R., and Balogh, A.: Production of gyrating ions from nonlinear wave-particle interaction upstream from the Earth's bow shock: a case study from Cluster-CIS, submitted to Planet. Space Sci., 2002.

Motschmann, U., Woodward, T. I., Glassmeier, K.-H., Southwood, D. J., and Pinçon,J. L.: Wavelength and direction filtering by magnetic measurements at satellite arrays: Generalized minimum variance analysis, J. Geophys. Res., 101, 4961-4965, 1996.

Neubauer, F. M. and Glassmeier, K.-H.: Use of an Array of Satellites as a Wave Telescope, J. Geophys. Res., 95, 19 115-19 122, 1990.

Neubauer, F. M., Glassmeier, K.-H., Walter, R., and Dunlop, M.: Cluster as a Wave Telescope, in: Proc. of the International Workshop on "Space Plasma Physics Investigations by Cluster and Regatta”, ESA SP-306, European Space Agency, Paris, 51-55, 1990.

Paschmann, G. and Daly, P. W. (Eds): Analysis methods for multispacecraft data, International Space Science Institute, Bern, 1998.

Pillai, S. U.: Array Signal Processing, Springer-Verlag, New York, 1989.

Pinçon, J. L., Lefeuvre, F., and Parrot, M.: Experimental Constraints in the Characterization of a Turbulent Field from Several Satellites, in: Proc. of the International Workshop on "Space Plasma Physics Investigations by Cluster and Regatta”, ESA SP306, European Space Agency, Paris, 57-67, 1990.

Pinçon, J.-L. and Motschmann, U.: Multi-Spacecraft Filtering: General Framework in Analysis methods for multi-spacecraft data, (Eds) Paschmann, G. and Daly, P. W., International Space Science Institute, Bern, 65-78, 1998.

Rème, H., Aoustin, C., Bosqued, J. M., Dandouras, I., Lavraud, B., Sauvaud, J. A., Barthe, A., Bouyssou, J., Camus, T., Coeur-Joly, O., Cros, A., Cuvilo, J., Ducay, F., Garbarowitz, Y., Medale, J. L., Penou, E., Perrier, H., Romefort, D., Rouzaud, J., Vallat, C., Alcaydé, D., Jacquey, C., Mazelle, C., d'Uston, C., Möbius, E., Kistler, L. M., Crocker, K., Granoff, M., Mouikis, C., Popecki, M., Vosbury, M., Klecker, B., Hovestadt, D., Kucharek, H., Kuenneth, E., Paschmann, G., Scholer, M., Sckopke, N., Seidenschwang, E., Carlson, C. W., Curtis,D. W., Ingraham, C., Lin, R. P., McFadden, J. P., Parks, G. K., Phan, T., Formisano, V., Amata, E., Bavassano-Cattaneo, P., Baldetti, M. B., Bruno, R., Chionchio, G., Di Lellis, A., Marcucci, M. F., Pallocchia, G. Korth, A., Daly, P. W., Graeve, B., Rosenbauer, H., Vasyliunas, V., McCarthy, M., Wilber, M., Eliasson, L., Lundin, R., Olsen, S., Shelley, E. G., Fuselier, S., Ghielmetti, A. G., Lennartsson, W., Escoubet, C. P., Balsiger, H., Friedel, R., Cao, J.-B., Kovrazhkin, R. A., Papamastorakis,I., Pellat, R., Scudder, J., and Sonnerup, B. U. O̊: First multispacecraft ion measurements in and near the Earth's magnetosphere with identical Cluster Ion Spectrometry (CIS) experiment, Ann. Geophysicae, 19, 1303-1354, 2001. 
Schwartz, S. J. and Burgess, D.: Quasi-Parallel Shocks: A Patchwork of Three-Dimensional Structures, Geophys. Res. Lett., 18, 373-376, 1991.

Schwartz, S. J., Burgess, D., Wilkinson, W. P., Kessel, R. L., Dunlop,M. W., and Luhr, H.: Observations of Short Large-Amplitude Magnetic Structures at a Quasi-Parallel Shock, J. Geophys. Res., 97, 4209-4227, 1992.
Sentman, D. D., Edmiston, J. P., and Frank, L. A.: Instabilities of Low Frequency, Parallel Propagating Electromagnetic Waves in the Earth's Foreshock Region, J. Geophys. Res., 86, 7487-7497, 1981.

Song, P. and Russell, C. T.: Time Series Data Analysis in Space Physics, Space. Sci. Rev., 87, 387-463, 1999. 